Anais da Academia Brasileira de Ciências (2003) 75(1): 1-7

(Annals of the Brazilian Academy of Sciences)

ISSN 0001-3765

www.scielo.br/aabc

\title{
On four dimensional Dupin hypersurfaces in Euclidean space
}

\author{
CARLOS M. C. RIVEROS and KETI TENENBLAT \\ Departamento de Matemática, IE.UNB, 70910-900 Brasília, Brazil \\ Manuscript received on August 8, 2002; accepted for publication on October 28, 2002; \\ contributed by Keti TenENBLAT*
}

\begin{abstract}
Dupin hypersurfaces in five dimensional Euclidean space parametrized by lines of curvature, with four distinct principal curvatures, are considered. A generic family of such hypersurfaces is locally characterized in terms of the principal curvatures and four vector valued functions of one variable. These functions are invariant by inversions and homotheties.
\end{abstract}

Key words: Dupin hypersurfaces, Laplace invariants, lines of curvature, principal curvatures.

\section{INTRODUCTION}

Dupin surfaces were first studied by Dupin in 1822 and more recently by many authors (Riveros and Tenenblat preprint, Cecil and Chern 1989, Cecil and Jensen 1998, 2000, Miyaoka 1984, Niebergall 1992, Pinkall 1985a,b, Stolz 1999 and Thorbergsson 1983), which studied several aspects of Dupin hypersurfaces. The class of Dupin hypersurfaces is invariant under conformal transformations. Moreover, the Dupin property is invariant under Lie transformations (Pinkall 1985b). Therefore, the classification of Dupin hypersurfaces is considered up to these transformations. The local classification of Dupin hypersurfaces is considered up to these transformations. The local classification of Dupin surfaces in $\mathbb{R}^{3}$ is well known. Pinkall (1985a) gave a complete classification up to Lie equivalence for Dupin hypersurfaces $M^{3} \subset \mathbb{R}^{4}$. However, the classification of Dupin hypersurfaces for higher dimensions is far from complete. Therefore, it is important to characterize such submanifolds. Our main result, Theorem 5, provides a local characterization of generic Dupin hypersurfaces in $\mathbb{R}^{5}$, with four distinct principal curvatures. The details of the proof can be found in (Riveros and Tenenblat preprint) and they will appear elsewhere. The proof is based on the theory of higher-dimensional Laplace invariants, which we recall in section 2, and the properties of Dupin hypersurfaces with distinct principal curvatures, given in section 3.

\footnotetext{
* Member of Academia Brasileira de Ciências

Correspondence to: Keti Tenenblat

E-mail: keti@mat.unb.br
} 


\section{THE HIGHER-DIMENSIONAL LAPLACE INVARIANTS}

The results in this section were obtained by Kamram and Tenenblat $(1996,1998)$.

We consider linear systems of second-order partial differential equations, of the form

$$
Y_{, k l}+a_{k l}^{k} Y_{, k}+a_{k l}^{l} Y_{, l}+c_{k l} Y=0, \quad 1 \leq k \neq l \leq n,
$$

where $Y$ is a scalar function of the independent variables $x_{1}, x_{2}, \ldots, x_{n}, Y_{, l}$ denotes the derivative of $Y$ with respect to $x_{l}$ and the coefficients $a$ and $c$ are smooth functions of $x_{1}, x_{2}, \ldots, x_{n}$ which are symmetric in the pair of lower indices and satisfy certain compatibility conditions. The general form of the system (1) is preserved under admissible transformations

$$
\begin{aligned}
& Y=\varphi\left(x_{1}, x_{2}, \ldots, x_{n}\right) \bar{Y}, \\
& x_{1}=f_{i}\left(\bar{x}_{i}\right), \quad 1 \leq i \leq n,
\end{aligned}
$$

where $\varphi$ is smooth and non-vanishing and the $f_{i}$ 's are smooth and have non-vanishing derivatives. It is easily verified that under an admissible transformation, the coefficients $a$ and $c$ transform according to,

$$
\begin{aligned}
& \bar{a}_{l k}^{l}=f_{k}^{\prime}\left[a_{k l}^{l}+(\log \varphi)_{, k}\right], \\
& \bar{c}_{l k}=f_{l}^{\prime} f_{k}^{\prime}\left[c_{l k}+a_{l k}^{l}(\log \varphi)_{, l}+a_{l k}^{k}(\log \varphi)_{, k}+\frac{\varphi_{, k l}}{\varphi}\right], \quad 1 \leq k \neq l \leq n,
\end{aligned}
$$

and the system (1) is

$$
\bar{Y}_{, k l}+\bar{a}_{k l}^{k} \bar{Y}_{, k}+\bar{a}_{k l}^{l} \bar{Y}_{, l}+\bar{c}_{k l} \bar{Y}=0, \quad 1 \leq k \neq 1 \leq n
$$

The higher-dimensional Laplace invariants of (1) are defined to be the $n(n-1)^{2}$ functions given by

$$
m_{i j}=a_{i j, i}^{i}+a_{i j}^{i} a_{i j}^{j}-c_{i j}, \quad m_{i j k}=a_{k j}^{k}-a_{i j}^{i}, \quad k \neq i, j
$$

for all ordered pairs $(i, j), 1 \leq i \neq j \leq n$.

Lemma 1. The higher-dimensional Laplace invariants of a compatible system (1) satisfy the following relations:

$$
\begin{aligned}
m_{i j k}+m_{k j i} & =0, \\
m_{i j k, k}-m_{i j k} m_{j k i}-m_{k j} & =0, \\
m_{i j, k}+m_{i j k} m_{i k}+m_{i k j} m_{i j} & =0, \\
m_{i j k}-m_{i j l}-m_{l j k} & =0, \\
m_{l i k, j}+m_{i j l} m_{k i l}+m_{l j k} m_{k i j} & =0 .
\end{aligned}
$$

for $1 \leq i, j, k, l \leq n, \quad i, j, k, l$ distinct. 
The functions $m_{i j}, m_{i j k}$ are invariant under pure rescalings (2). The expression of a system (1) in terms of its higher-dimensional Laplace invariants is established in the following Theorem. For proof and details we refer also to (Tenenblat 1998).

THEOREM 2. Given any collection of $n(n-1)^{2}, n \geq 3$, smooth functions of $x_{1}, x_{2}, \ldots, x_{n}$ $m_{i j}, m_{i j k}, 1 \leq i, j, k \leq n, i, j, k$ distinct, satisfying the constraints (4), there exists a linear system (1) whose higher-dimensional Laplace invariants are the given functions $m_{i j}, m_{i j k}$. Any such system is defined up to rescaling (2). A representative is given by

$$
\begin{aligned}
Y_{, i j}+A Y_{, j}-m_{i j} Y & =0, \\
Y_{, i k}+\left(A+m_{j i k}\right) Y_{, k}-m_{i k} Y & =0, \\
Y_{, j k}+m_{i k j} Y_{, j}+m_{i j k} Y_{, k} & =0, \\
Y_{, l k}+m_{i k l} Y_{, l}+m_{i l k} Y_{, k} & =0 .
\end{aligned}
$$

where $(i, j)$ is a fixed (ordered) pair, $1 \leq i, j, k, l \leq n$ are distinct and $A$ is a function which satisfies the following:

$$
A_{, j}=m_{j i}-m_{i j}, \quad A_{, k}=-m_{j k i, i}
$$

\section{DUPIN HYPERSURFACES WITH DISTINCT PRINCIPAL CURVATURES}

Definition 3. An immersion $X: \Omega \subset \mathbb{R}^{n} \rightarrow \mathbb{R}^{n+1}$ is called a Dupin hypersurface if along each curvature line the corresponding principal curvature is constant.

Let $X: \Omega \subset \mathbb{R}^{n} \rightarrow \mathbb{R}^{n+1}$ be a Dupin hypersurface parametrized by lines of curvature, with distinct principal curvatures $\lambda_{i}, 1 \leq i \leq n$ and le $N: \Omega \subset \mathbb{R}^{n} \rightarrow \mathbb{R}^{n+1}$ be a unit vector field normal to $X$. Then

$$
\begin{aligned}
\left\langle X_{, i}, X_{, j}\right\rangle & =\delta_{i j} g_{i i}, 1 \leq i \leq n, \\
N_{, i} & =\lambda_{i} X_{, i}, 1 \leq i \leq n \\
\lambda_{i, i} & =0,1 \leq i \leq n .
\end{aligned}
$$

Moreover

$$
\begin{aligned}
& X_{, i j}-\Gamma_{i j}^{i} X_{, i}-\Gamma_{i j}^{j} X_{, j}=0,1 \leq i \neq j \leq n, \\
& \Gamma_{i j}^{i}=\frac{\lambda_{i, j}}{\lambda_{j}-\lambda_{i}}, 1 \leq i \neq j \leq n,
\end{aligned}
$$

where $\Gamma_{i j}^{k}$ are the Christoffel symbols.

As a consequence of the relations (3) and Lemma 1, we obtain the following expressions for the higher-dimensional Laplace invariants

$$
\begin{aligned}
m_{i j} & =-\Gamma_{i j, i}^{i}+\Gamma_{i j}^{i} \Gamma_{i j}^{j}, \\
m_{i j k} & =\Gamma_{i j}^{i}-\Gamma_{k j}^{k} .
\end{aligned}
$$


Moreover, for $1 \leq i, j, k, l \leq n, i, j, k, l$ distinct, one has

$$
\begin{aligned}
m_{i j} & =0, \\
m_{i j k}+m_{k j i} & =0, \\
m_{i j k, k}-m_{i j k} m_{j k i} & =0, \\
m_{i j k}-m_{i j l}-m_{l j k} & =0, \\
m_{l i k, j}+m_{i j l} m_{k i l}+m_{l j k} m_{k i j} & =0 .
\end{aligned}
$$

Let $X: \Omega \subset \mathbb{R}^{n} \rightarrow \mathbb{R}^{n+1}, n \geq 3$, be a Dupin hypersurface parametrized by lines of curvature, with distinct principal curvatures. Consider a homothety

$$
\begin{aligned}
D: \mathbb{R}^{n+1} & \rightarrow \mathbb{R}^{n+1} \\
X & \rightarrow D(X)=a X, a \in \mathbb{R}, a \neq 0
\end{aligned}
$$

and assuming $0 \notin X(\Omega)$ an inversion

$$
\begin{aligned}
I^{n+1}: \mathbb{R}^{n+1}-\{0\} & \rightarrow \mathbb{R}^{n+1}-\{0\} \\
X & \rightarrow I^{n+1}(X)=\frac{X}{\langle X, X\rangle} .
\end{aligned}
$$

Let $Y=I^{n+1}(X)$ and $\bar{Y}=D(X)$. Then the higher-dimensional Laplace invariants of $Y$ and $\bar{Y}$ are those of $X$, since inversions and homotheties are rescalings (2).

The next result is an application of Theorem 2 .

Lemma 4. Let $X: \Omega \subset \mathbb{R}^{n} \rightarrow R^{n+1}, n \geq 3$, be a Dupin hypersurface parametrized by lines of curvature and $\lambda_{r}, 1 \leq r \leq n$, distinct principal curvatures of each point. For $i, j, k$ fixed, $1 \leq i \neq j \neq k \leq n$, the transformation

$$
X=V \bar{X}, \quad \text { with } \quad V=\frac{e^{\int \frac{\lambda k-\lambda j}{\lambda_{j}-\lambda_{i}} m_{j k i} d x_{k}}}{\lambda_{j}-\lambda_{i}},
$$

transforms the system (5) into

$$
\begin{aligned}
\bar{X}_{, i j}+A \bar{X}_{, j} & =0, \\
\bar{X}_{, i r}+\left(A+m_{j i r}\right) \bar{X}_{, r} & =0, \\
\bar{X}_{, j r}+m_{i r j} \bar{X}_{, j}+m_{i j r} \bar{X}_{, r} & =0, \\
\bar{X}_{, r l}+m_{i l r} \bar{X}_{, r}+m_{i r l} \bar{X}_{, l} & =0,
\end{aligned}
$$

where $l \neq r$ are distinct from $i, j$,

$$
A=-\int m_{j k i, i} d x_{k}
$$

and

$$
A_{, j}=0, A_{, r}=-m_{j r i, i}
$$




\section{A CHARACTERIZATION OF DUPIN HYPERSURFACES IN $\mathbb{R}^{5}$}

We can now state our main result which provides a local characterization for generic Dupin hypersurfaces in $\mathbb{R}^{5}$, with four distinct curvatures. Considering the functions $m_{i j k}$ satisfying (6), we introduce the following functions admitting that $m_{213} \neq 0, m_{214} \neq 0$ and $m_{314} \neq 0$,

$$
\begin{gathered}
T=m_{214}+\left[\log \left(\frac{m_{213}}{m_{314}}\right)\right]_{, 1} \\
U=m_{314}+\left[\log \left(\frac{m_{213}}{m_{314}}\right)\right]_{, 1} \\
P_{1}^{4}=m_{213} T, \quad P_{2}^{4}=m_{213} U, \quad P_{3}^{4}=m_{214} U .
\end{gathered}
$$

TheOREM 5. Let $X: \Omega \subset \mathbb{R}^{4} \rightarrow \mathbb{R}^{5}$, be a Dupin hypersurface parametrized by lines of curvature, with principal curvatures, $\lambda_{i}, 1 \leq i \leq 4$, distinct at each point. Suppose $m_{234} \neq 0, m_{423} \neq 0$, $P_{i}^{4} \neq 0$, for all $i, 1 \leq i \leq 3$, then

$$
X=V\left[\sum_{i=1}^{3}(-1)^{i+1} B_{i}^{4}\right]
$$

where

$$
V=\frac{e^{\int \frac{\lambda_{3}-\lambda_{2}}{\lambda_{2}-\lambda_{1}} m_{231} d x_{3}}}{\lambda_{2}-\lambda_{1}}, B_{i}^{4}=\frac{1}{Q_{i}^{4}}\left[\int \frac{Q_{i}^{4} G_{1}\left(x_{1}\right)}{P_{i}^{4}} d x_{1}+G_{i+1}\left(x_{i+1}\right)\right], 1 \leq i \leq 3,
$$

$P_{i}^{4}, 1 \leq i \leq 3$, are defined by (10), $G_{i}\left(x_{i}\right), 1 \leq i \leq 4$, are vector valued functions of $\mathbb{R}^{5}, A=$ $-\int m_{231,1} d x_{3}$ and

$$
Q_{i}^{4}=\left\{\begin{array}{l}
e^{\int A d x_{1}} \quad \text { if } \quad i=1 \\
e^{\int\left(A+m_{21(i+1)}\right) d x_{1}} \quad \text { if } \quad 2 \leq i \leq 3
\end{array}\right.
$$

Moreover, considering

$$
\alpha^{1}=\left(A+\frac{\lambda_{2,1}}{\lambda_{1}-\lambda_{2}}\right) M+M_{, 1}, \quad \alpha^{j}=\frac{\lambda_{1, j}}{\lambda_{j}-\lambda_{1}} M+M_{, j}, \quad 2 \leq j \leq 4,
$$

where $M=\sum_{i=1}^{3}(-1)^{i+1} B_{i}^{4}$, the functions $G_{i}\left(x_{i}\right)$ satisfy the following properties in $\Omega$, for all $1 \leq i, j \leq 4$ :

a) $\alpha^{i} \neq 0$

b) $\left\langle\alpha^{i}, \alpha^{j}\right\rangle=0, i \neq j$, 
c) $\lambda_{i}=-\frac{\left\langle\alpha_{, i}^{i}, \alpha^{1} \times \alpha^{2} \times \alpha^{3} \times \alpha^{4}\right\rangle}{V\left|\alpha^{i}\right|^{2}\left|\alpha^{1}\right|\left|\alpha^{2}\right|\left|\alpha^{3}\right|\left|\alpha^{4}\right|}$.

Conversely, let $\lambda_{i}: \Omega \subset \mathbb{R}^{4} \rightarrow \mathbb{R}, 1 \leq i \leq 4$ be real functions distinct at each point, such that $\lambda_{i, i}=0$, and the functions $m_{i j k}$, defined by

$$
m_{i j k}=\frac{\lambda_{i, j}}{\lambda_{j}-\lambda_{i}}-\frac{\lambda_{k, j}}{\lambda_{j}-\lambda_{k}}, 1 \leq i \neq j \neq k \leq 4 .
$$

satisfy (6). Then for any vector valued functions $G_{i}\left(x_{i}\right)$ satisfying the properties $\left.\left.a\right) b\right) c$ ) above, where $\alpha^{i}$ are defined by (14), the application $X: \Omega \subset \mathbb{R}^{4} \rightarrow \mathbb{R}^{5}$ given by (11) describe a Dupin hypersurface parametrized by lines of curvature whose principal curvatures are $\lambda_{i}$.

\section{SKETCH OF THE PROOF}

Let $X$ be a Dupin hypersurface as in Theorem 5 then it follows from Lemma 4 that

$$
X=\frac{V}{m_{213}}\left[W^{3}-W^{2}\right]
$$

where $V$ is given by (12) and the vector valued functions $W^{3}\left(x_{1}, x_{2}, x_{4}\right)$ and $W^{2}\left(x_{1}, x_{3}, x_{4}\right)$ satisfy systems of differential equations of Laplace type. The solutions of the systems are given by

$$
\begin{aligned}
W^{3} & =\frac{m_{213}}{Q_{1}^{4}}\left[\int \frac{Q_{1}^{4} G_{1}\left(x_{1}\right)}{P_{1}^{4}} d x_{1}+G_{2}\left(x_{2}\right)\right]-\frac{m_{314}}{Q_{3}^{4}}\left[\int \frac{Q_{3}^{4} G_{1}\left(x_{1}\right)}{P_{3}^{4}} d x_{1}+G_{4}\left(x_{4}\right)\right] \\
W^{2} & =\frac{m_{213}}{Q_{2}^{4}}\left[\int \frac{Q_{2}^{4} F\left(x_{1}\right)}{P_{2}^{4}} d x_{1}+G_{3}\left(x_{3}\right)\right]-\frac{m_{214}}{Q_{3}^{4}}\left[\int \frac{Q_{3}^{4} F\left(x_{1}\right)}{P_{3}^{4}} d x_{1}+\bar{G}_{4}\left(x_{4}\right)\right]
\end{aligned}
$$

It can be shown that

$$
G_{1}\left(x_{1}\right)=F\left(x_{1}\right), \quad G_{4}\left(x_{4}\right)=\bar{G}_{4}\left(x_{4}\right)
$$

which proves (11).

The conditions a), b) of Theorem 5 are obtained considering that the vectors $X_{, i}$ are orthogonal and non-vanishing, condition c) is equivalent to requiring $\lambda_{i}$ to be principal curvature of $X$. The converse is a straightforward calculation.

REMARK. One can show that the vector valued functions $G_{i}\left(x_{i}\right)$ in Theorem 5 are invariant by inversions and homotheties of the corresponding Dupin hypersurfaces in $\mathbb{R}^{5}$. We conclude by mentioning that a characterization of a non-generic family of Dupin hypersurface $M^{n} \subset \mathbb{R}^{n+1}, n \geq$ 3 whose principal curvatures are distinct and $m_{i j k}=0, \forall i, j, k$ distinct has been obtained and it will appear elsewhere.

\section{ACKNOWLEDGMENTS}

The authors were partially supported by CNPq. 


\section{RESUMO}

Consideramos hipersuperfícies de Dupin no espaço Euclideano de dimensão cinco, parametrizadas por linhas de curvaturas, com quatro curvaturas principais distintas. Uma família genérica de tais hipersuperfícies é localmente caracterizada em termos das curvaturas principais e quatro funções vetoriais de uma variável. Essas funções são invariantes por inversões e homotetias.

Palavras-chave: hipersuperfícies de Dupin, invariantes de Laplace, linhas de curvatura, curvaturas principais.

\section{REFERENCES}

Cecil T and Chern SS. 1989. Dupin submanifolds in Lie sphere geometry. Differential geometry and topology 1-48. Lecture Notes in Math 1369, Springer.

Cecil T and Jensen G. 1998. Dupin hypersurfaces with three principal curvatures. Invent Math 132: 121-178.

Cecil T and Jensen G. 2000. Dupin hypersurfaces with four principal curvatures. Geom Dedicata 79: $1-49$.

Kamran N and Tenenblat K. 1996. Laplace transformation in higher dimensions. Duke Math J 84: 237-266.

Kamran N and Tenenblat K. 1998. Periodic systems for the higher-dimensional Laplace transformation. Discrete and continuous dynamical systems, Vol. 4: 359-378.

MiYaоKa R. 1984. Compact Dupin hypersurfaces with three principal curvatures. Math Z 187: 433-452.

Niebergall R. 1992. Dupin hypersurfaces in $\mathbb{R}^{5}$. Geom Dedicata 41: 5-38.

PinKall U. 1985a. Dupinsche Hyperflachen in $E^{4}$. Manuscripta Math 51: 89-119.

Pinkall U. 1985b. Dupin hypersurfaces. Math Ann 270: 427-440.

Riveros CMC and Tenenblat K. Dupin hypersurfaces in $\mathbb{R}^{5}$ with four distinct principal curvatures, preprint, Universidade de Brasília.

Stolz S. 1999. Multiplicities of Dupin hypersurfaces. Invent Math 138: 253-279.

Tenenblat K. 1998. Transformations of Manifolds and Applications to Differential Equations. Addison Wesley Longman Inc.

Thorbergsson G. 1983. Dupin hypersurfaces. Bull London Math Soc 15: 493-498. 